\title{
Optimising outcomes: socioeconomic perspective
}

\author{
D B Mark
}

All medical interventions carry costs, and the economic implications are a major consideration when any change in medical practice is proposed. New drugs are often more expensive than those that they are designed to replace, and the introduction of new procedures may also entail increased costs. Medical costs include not only the cost of the drug itself, but also the induced costs (or sometimes, cost savings) such as the costs of administering the drug and the consequent levels of use of other medical resources, including medical personnel. The ESSENCE study (Efficacy and Safety of Subcutaneous Enoxaparin in Non-Q-wave Coronary Events) - a double blind, randomised trial of the low molecular weight heparin enoxaparin and unfractionated heparin $(\mathrm{UFH})$ in unstable angina and non-Q wave myocardial infarction ${ }^{1}$-addressed this issue by means of a prospectively designed economic study to evaluate the cost implications in the light of clinical outcomes. ${ }^{2}$ The ESSENCE economic study was undertaken on those patients in the US, and examined both the use of medical resources and the medical costs to 30 days.

\section{Methods}

The case report form used in the ESSENCE study was designed to include information on use of medical resources. Full medical billing information was available for 655 patients out of the total of 923 enrolled in the US, and a conversion factor included in each hospital's Medicare cost report was used to convert the charges to costs. The national Medicare fee schedule was used to assign physician fees for the purposes of this analysis. Billing information was not available for 268 patients; in these cases costs were imputed using a statistical regression model developed from those patients from whom billing information was available. Details of the costs applied are shown in table 1. For the purposes of this analysis, costs associated with the time spent in hospital before randomisation (less than one day) were excluded, as were any costs associated with hospitalisation more than 30 days after the index event.

and Assessment

Group, Department of

Medicine,

Cardiovascular

Division, Box 3485,

Duke University

Medical Center,

Durham, NC 27710,

USA

D B Mark

Correspondence to: Dr Mark.
Table 1 Costs applied in hospital costs regression model

\begin{tabular}{lc}
\hline Resource & Cost (US\$) \\
\hline Intensive care unit, per day & 1506 \\
Hospital stay, non-intensive care, per day & 761 \\
Diagnostic catheterisation & 1197 \\
Percutaneous transluminal coronary angioplasty & 3773 \\
Coronary artery bypass grafting & 8522 \\
\hline
\end{tabular}

\section{Results}

DRUG COSTS

Enoxaparin was administered as a twice daily subcutaneous injection, using multidose vials to keep waste to a minimum. Drug cost was calculated at US $\$ 69$ per day. Mean duration of enoxaparin treatment was 2.25 days, bringing the total drug costs in this arm of the trial to US $\$ 155$

While UFH costs less than enoxaparin, it is also more complicated to administer and those additional costs must be considered. For this analysis, the cost of the drug itself, its preparation in the hospital pharmacy, rental of an infusion pump for the duration of treatment, and the costs of activated partial thromboplastin time monitoring were all included. Treatment with intravenous UFH also entails physician and nursing time to monitor and adjust the infusion, but these costs were not included in the analysis because it was not clear that they were recoverable if UFH was not used. On this basis, the total cost of heparin for the mean treatment period of 2.5 days was US $\$ 80$, US $\$ 75$ less than enoxaparin treatment.

USE OF HOSPITAL RESOURCES

In the trial as a whole, the length of the initial stay in the intensive care unit was 0.2 days shorter for patients treated with enoxaparin. In the US cohort the stay was 0.3 days shorter for patients treated with enoxaparin (fig 1). Although this difference is not significant, it should be borne in mind that ESSENCE was a double blind trial so the slightly shorter stay occurred without the physicians being aware of the treatment patients had received. The total length of the initial hospital stay, the total days in intensive care to 30 days, and the total hospital stay to 30 days all showed a difference between the two treatment groups with a trend favouring enoxaparin over UFH.

Diagnostic cardiac catheterisation was performed during the initial hospital stay in more patients in the US cohort than in the study

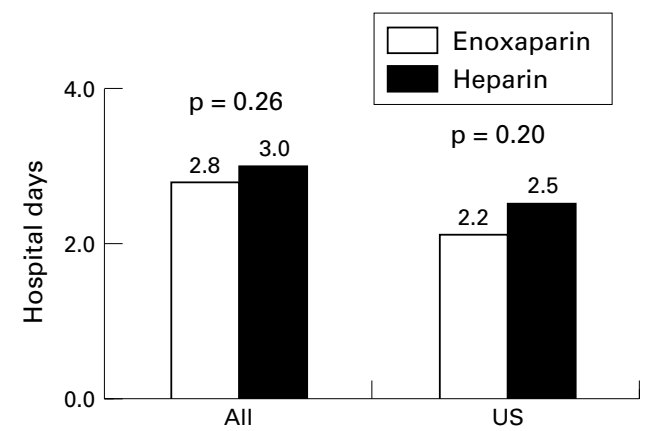

Figure 1 Length of initial stay in intensive care unit (days). 


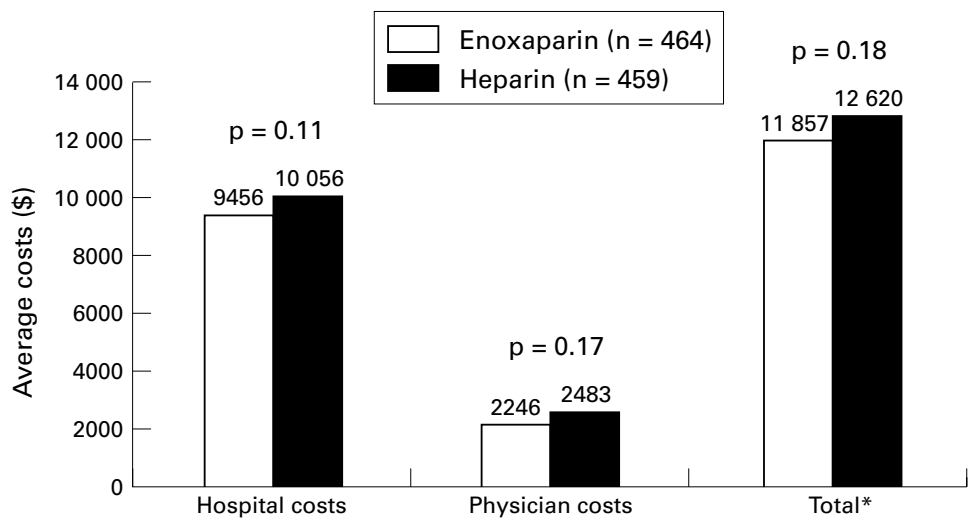

Observed costs data $(n=655)+$ imputed costs $(n=268)$ * Includes costs of enoxaparin

Figure 2 Medical costs for US cohort during initial hospital stay.

patients as a whole. However, in both the US cohort and the entire study cohort, there was a trend for lower rates of catheterisation in the patients receiving enoxaparin than in the patients receiving UFH $(53 \%$ v $57 \%$ US cohort, $43 \% v 46 \%$ total cohort).

The overall ESSENCE results showed a significant reduction in the rate of percutaneous transluminal coronary angioplasty during the first 30 days with enoxaparin, from $18 \%$ to $14 \%(\mathrm{p}=0.003)$. Although US intervention rates are generally higher than in the total cohort, a trend towards a reduction in the rate of coronary angioplasty to 30 days, from $22 \%$ with UFH to $18 \%$ with enoxaparin $(\mathrm{p}=0.08)$, was still apparent. Coronary artery bypass grafting rates during the initial hospital stay were the same in both treatment groups in the total cohort, whereas in the US cohort there was a slight reduction from $14 \%$ to $12 \%$ for patients treated with enoxaparin. A trend of a reduced rate of bypass grafting was also apparent in the US cohort at 30 days (14\% v 17\%, $\mathrm{p}=0.15)$.

COST IMPLICATIONS OF RESOURCE USE During the initial hospital stay, enoxaparin treatment was associated with a cost saving of US $\$ 763$ per patient (fig 2). This figure includes the additional cost of US $\$ 75$ for

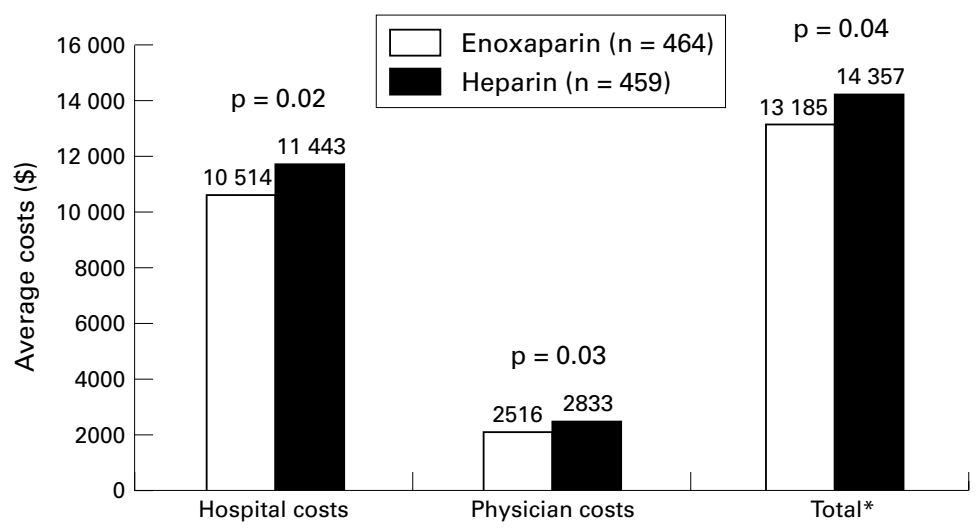

Observed costs data $(n=655)+$ imputed costs $(n=268)$ *Includes costs of enoxaparin

Figure 3 Medical costs for US cohort to 30 days. enoxaparin treatment, and a saving of US $\$ 237$ on physician fees and US $\$ 600$ in hospital costs.

At 30 days, the difference between UFH and enoxaparin is larger than at the end of the initial hospital stay (fig 3). The absolute cost saving with enoxaparin at 30 days was US $\$ 1172$, including the cost of purchasing enoxaparin, and this difference between the two groups is significant $(\mathrm{p}=0.04)$.

\section{Discussion}

The clinical outcomes of the ESSENCE study showed that enoxaparin offers clinical advantages over UFH at 14 and 30 days, with a similar safety record. ${ }^{1}$ Data from the one year follow up analysis is now also available, ${ }^{3}$ and shows that these benefits are maintained over the long term. However, effective treatments are of little value if health care systems cannot afford to use them. The purchase price of UFH is very low, whereas the newer low molecular weight heparins all tend to be more expensive. This study has shown that despite a higher purchase price, the better clinical outcomes resulting from treatment with enoxaparin result in lower overall costs.

The economic study was undertaken on the 936 patients enrolled in ESSENCE in the US, who represent only a proportion of the total study of 3171 patients. Standard medical practice varies between countries, and these differences are apparent in the ESSENCE results. The US tends to have higher rates of medical intervention than many other countries, and this is apparent in the rates of diagnostic catheterisation and revascularisation procedures reported here. However, a consistent picture emerges of a reduced need for procedures of all kinds and a slightly shorter hospital stay with enoxaparin, and this was apparent in the trial as a whole as well as in the US cohort.

Variations in relative costs and differences in medical practice mean that the results reported here cannot be directly applied to other countries, or to the ESSENCE trial as a whole. However, it seems likely that economic analyses performed elsewhere would produce a similar picture of the higher cost of enoxaparin being more than compensated for by the cost savings implicit in a lower clinical event rate. When a treatment is associated with better outcomes and lower costs, it is described by economists as the "dominant" treatment, meaning that from a policy point of view, it is most likely preferable to the treatment it has improved upon.

\section{Conclusions}

Compared with patients receiving standard treatment with UFH, enoxaparin patients had consistently lower lengths of hospital stay, cardiac procedure rates, and lower costs to 30 days for both hospital treatment and physician time. These benefits meant that in the US cohort of patients, enoxaparin was an economically attractive treatment, and was associated with an absolute cost saving of over US $\$ 1000$ per patient. 
1 Cohen M, Demers C, Gurfinkel EP, for the ESSENCE Study Group. A comparison of low-molecular-weight heparin with unfractionated heparin for unstable coronary artery disease: efficacy and safety of subcutaneous enoxaparin in non-Q-wave cor

Mark DB, Cowper PA, Berkowitz S, et al. Economic assessment of low molecular weight heparin (enoxaparin) versus unfractionated heparin in acute coronary syndromes patients: results from the ESSENCE randomized trial.
Efficacy and safety of subcutaneous enoxaparin in non- $Q$ wave coronary events [unstable angina or non-Q-wave myocardial infarction]. Circulation 1998;97:1702-7.

3 Goodman S, Bigonzi F, Radley D, et al, for the ESSENCE Study Group. One-year follow-up of the ESSENCE trial (enoxaparin versus heparin in unstable angina and non-Qwave myocardial infarction). XXth Congress of the European Society of Cardiology, Vienna, Austria, 22-26 August 1998 [abstract]. Eur Heart f 1998;19(suppl):P477. 